\title{
Functional consequences of chromosomal rearrangements on gene expression: not so deleterious after all?
}

\author{
Yad Ghavi-Helm \\ Institut de Génomique Fonctionnelle de Lyon, Univ Lyon, CNRS UMR 5242, Ecole Normale \\ Supérieure de Lyon, Université Claude Bernard Lyon 1, 46 allée d'Italie F-69364 Lyon, France \\ Correspondence to: \\ email: yad.ghavi-helm@ens-lyon.fr / phone:+33 426731350
}

Keywords (5): chromatin organization; chromosomal rearrangements; topologically associated domains; enhancers; transcription

\section{Research Highlights:}

- TADs are evolutionarily conserved regions largely overlapping with regulatory domains

- Deleting TAD boundaries only mildly affects gene expression

- Chromosomal inversions affecting TAD boundaries are usually more deleterious

- Enhancer redundancy and enhancer-promoter specificity might buffer the effect of chromosomal rearrangements

Declarations of interest: none 


\section{Abstract (max 250 words)}

Chromosomes are folded and organized into topologically associated domains (TADs) which provide a framework for the interaction of enhancers with the promoter of their target gene(s). Structural rearrangements observed during evolution or in disease contexts suggest that changes in genome organization strongly affect gene expression and can have drastic phenotypic effects. In this review, I will discuss how recent genomic engineering experiments reveal a more contrasted picture, suggesting that TADs are important but not always essential for gene expression regulation. 


\section{Introduction}

In metazoans, the expression of protein-coding genes is finely regulated both in space and time through the action of hundreds of thousands of regulatory elements, such as enhancers, scattered along the non-coding genome. Enhancers are short genomic regions containing DNA motifs, which act as binding sites for transcription factors. When ectopically inserted into the genome, enhancers can drive the expression of a reporter gene in an orientation- and context-independent manner ${ }^{1,2}$. Active enhancers are located in open chromatin regions ${ }^{3}$ and are characterized by the presence of specific chromatin marks such as $\mathrm{H} 3 \mathrm{KK} 4 \mathrm{me} 1$ and $\mathrm{H} 3 \mathrm{~K} 27 \mathrm{ac}^{4-8}$. Importantly, enhancers can function either in an additive or partially redundant manner, collectively establishing the complex spatiotemporal expression pattern of their target gene $(s)^{9}$. A particularly intriguing feature of enhancers is their ability to operate at a distance: they can be located at great linear distances from their target gene(s) but yet activate their expression via the formation of chromatin loop $(\mathrm{s})^{9}$. While enhancerpromoter looping is essential to activate gene expression, enhancers can sometimes interact with gene promoters in tissues or at developmental stages when the gene is not active ${ }^{10-13}$, suggesting that preformed topologies might enable rapid and efficient transcriptional activation. Even more intriguing, a given enhancer might regulate the expression of a distal gene while skipping other genes located in its proximity ${ }^{14}$. This reflects a certain degree of specificity in enhancer-promoter interactions: the expression of a gene cannot be activated by just any enhancer. The exact mechanisms controlling this enhancer-promoter interaction specificity remain however largely elusive. While multiple factors are likely involved (reviewed by van Arensbergen et al. ${ }^{15}$ ), the evolutionary conservation of regulatory domains and the transcriptional consequences of their disruption during disease suggest that the three-dimensional (3D) organization of the genome might be of particular importance. These observations are however challenged by the advent of genome engineering techniques, allowing the engineering of very precise and focused de-novo rearrangements such as deletions and inversions. This review illustrates how these recent results are refining our perception of the link between genome topology, enhancer-promoter interactions, and gene expression.

\section{D genome organization}

\section{Chromatin structure: compartments, TADs and loops}

To fit into the tiny cell nucleus, chromatin fibers need to be tightly folded. Classical 3D DNA fluorescent in situ hybridization (FISH) ${ }^{16}$ and the more recent Chromosome Conformation Capture (3C) technique and its derivatives (such as $\mathrm{Hi}-\mathrm{C}^{17}$ ) have provided increasing evidence suggesting that chromatin folding is both highly organized and tightly linked to transcriptional regulation. While this review primarily focuses on results obtained using 3C-derived approaches, direct visualization of chromatin organization in single cells has become a valuable alternative, thanks to the development of new methods and improvements in imaging resolution ${ }^{18-22}$ (reviewed by Cardozo Gizzi et al. in this issue). Chromosomal domains are positioned according to their transcriptional activity, with repressed regions often associated with the nuclear lamina ${ }^{23}$, while actively transcribed regions tend to be located at the center of the nucleus. Chromosomes are hierarchically organized into several types of domains. First, chromosomes can be divided into regions belonging to the $\mathrm{A}$ or $\mathrm{B}$ compartments, which broadly represent active and inactive chromatin respectively. These compartments can be identified by principal component analysis (PCA) of the normalized $\mathrm{Hi}-\mathrm{C}$ matrix and visualized as a checkerboard pattern in $\mathrm{Hi}-\mathrm{C} \mathrm{maps}^{17}$. The sign of the first eigenvector (principal 
component 1) indicates the compartment. This classification has subsequently been further refined in human and mouse cell lines into six sub-compartments (A1, A2, and B1 to B4) each associated with particular chromatin post-translational modifications ${ }^{24}$. At a smaller scale, chromosomes are divided into domains of increased 3D proximity ranging from about $100 \mathrm{~kb}$ to a few megabases in size, named Topologically Associating Domains (TADs) ${ }^{12,25,26}$. In vertebrates, the large majority of TADs are delimited by the binding of the CTCF insulator protein ${ }^{12,27}$ and have been proposed to form through a loop extrusion mechanism mediated by cohesin: the cohesin ring slides along the DNA until reaching two CTCF motifs positioned in a head-to-head orientation ${ }^{28-32}$. This can be visualized in $\mathrm{Hi}-\mathrm{C}$ maps by the presence of a strong signal (corner peak) at the tip of the TAD triangle, resulting from the high contact frequency between the CTCF-binding sites located at two adjacent TAD boundaries acting as loop anchors. However, it should be noted that not all loop anchors correspond to TAD boundaries, and that not all TAD domains display a strong corner peak ${ }^{24}$. Besides, some genomic regions, in particular gene-rich regions, are less structured and lack clear boundaries ${ }^{33}$, and a small proportion of TAD boundaries (10 to $20 \%$ ) are not bound by CTCF and might thus form in a CTCF-independent manner, possibly through other mechanisms than loop extrusion ${ }^{34}$. Accurately defining TADs is thus a complicated matter. A large variety of algorithms have been developed to identify TADs, resulting in a difference of up to one order of magnitude in the number of TADs called by different methods ${ }^{35,36}$. Because TADs adopt a hierarchical tree-like structure composed of nested triangles, small changes in the parameters used to identify them (such as sequencing depth, resolution or cut-off) can greatly influence their size. In addition, $\mathrm{Hi}$-C experiments themselves are not trivial: a variety of different protocols are available and reproducibility is a major issue. As a consequence, the structure and function of TADs and their potential role in transcriptional regulation are much debated.

TADs as functional regulatory domains?

It is generally admitted that TADs essentially restrict the activity of enhancers, preventing undesirable enhancer-promoter interactions across TAD boundaries. Indeed, the random insertion of a regulatory sensor acting as an enhancer-trap at various locations of the mouse genome revealed that the sensor only responds to the enhancers located within the same regulatory domain, and that these regulatory domains strongly correlate with $\operatorname{TADs}^{37}$. Besides, the genes and enhancers located within the same TAD tend to be co-regulated ${ }^{38-41}$. TADs can further be divided into "sub-TADs" or "sub-domains". While TADs are generally invariants between cell types, sub-TADs are cell-type specific and mediated by cohesin and Mediator ${ }^{27}$. Sub-TADs are also smaller (on average $32 \mathrm{~kb}$ versus $185 \mathrm{~kb}$ in lymphoblastoid cell lines ${ }^{42}$ ) and driven by transcription ${ }^{27}$. These initial studies suggest that TADs are functional regulatory units of the genome. However, recent evidence seems to contradict this view: TADs are lost upon CTCF or cohesin depletion but their loss only mildly affects gene expression ${ }^{34,43}$, questioning their actual role in transcriptional regulation. This result might however be specific to resting cells, as recent work has highlighted that cohesin is essential for inflammatory gene expression in primary myeloid cells ${ }^{44}$. A more direct method to establish TAD function is to examine the transcriptional consequences of their rearrangements, for example during evolution or in pathologies.

\section{Chromosomal rearrangements during evolution and in disease contexts}

Evolutionary conservation of TADs: proof of their importance or consequence of fragile TAD boundaries? 
A particularly strong argument in favor of a functional role for TADs is their evolutionary conservation: about $50 \%$ of TADs are conserved when comparing human and mouse $\mathrm{e}^{12,24}$. In fact, TADs generally overlap with syntenic blocks, i.e. regions of DNA with conserved collinearity of genes and/or non-coding elements, and syntenic rearrangement breakpoints are enriched at TAD boundaries both in vertebrates and flies ${ }^{45-49}$. However, this overlap might simply be the consequence of TAD boundaries' relative "fragility" to double-strand breaks (DSB). DSBs are often found at loop anchors, suggesting that loop extrusion might create some torsional stress subsequently released by topoisomerase II when the cohesin complex encounters a CTCF site ${ }^{50}$. In addition, TAD boundaries are enriched in open chromatin regions and active genes, which have an increased susceptibility to breakage. Damaged active genes tend to cluster in the nucleus ${ }^{51}$ where they get preferentially repaired with other regions in close 3D proximity. As a consequence, translocations tend to be enriched at TAD boundaries ${ }^{52-55}$. The remarkable evolutionary conservation of TADs also suggests that TAD boundary disruptions might be under purifying selection, i.e. that mutations affecting TAD boundaries are negatively selected during evolution. This is indeed the case: comparing human and ape populations revealed that deletions are depleted at TAD boundaries, but uniformly distributed in patients with developmental delay or autism ${ }^{56}$. This could however just be a consequence to the overall depletion of deletions at active genes which tend to be located at TAD boundaries.

\section{Chromosomal rearrangements in disease}

Another strong argument in favor of a functional role for TADs is the effect of structural rearrangements affecting TADs and causing various diseases, such as developmental disorders and cancers. This topic has already been extensively reviewed ${ }^{57-60}$, and is based on the following assumption: if TADs are required for proper enhancer-promoter communication, disturbing TADs by deletions ${ }^{61-65}$, duplications ${ }^{66}$, or inversions ${ }^{61}$ should have deleterious effects. A particularly striking example supporting this model came from the analysis of a genomic inversion affecting a TAD boundary at the Epha4 locus in mice. This inversion was shown to cause malformation syndromes in the developing limb bud due to the formation of de-novo interactions between Epha4 enhancers and the Wnt6 gene ${ }^{61}$. This mechanism, termed enhancer adoption ${ }^{67}$ or enhancer hijacking ${ }^{68}$, has been proposed to explain up to $12 \%$ of the deletions present in a database of rare genomic copynumber variants and their associated phenotypic data ${ }^{69}$. Similarly, TAD boundary disruptions have been linked to a variety of cancers ${ }^{68,70-73}$. For example, the expression of oncogenes in cancer cells can be upregulated due to the deletion or hypermethylation of cohesin and CTCF-binding sites at TADs boundaries ${ }^{71,73}$. Finally, a recent study uncovered that nearly all disease-associated short tandem repeats (daSTRs) are located at the boundaries of TADs, in particular those featuring a high density of $\mathrm{CpG}$ islands ${ }^{74}$.

These different examples suggest that altering TADs will necessarily cause adverse effects. Contrasting results have however emerged from two recent studies. First, Dong et al. compared the location of four translocations from the " 1000 genomes" project with TAD calls from the human IMR fibroblast cell line. They found that eight TADs were disrupted, altogether containing 34 genes. Out of these 34 genes, 16 were expressed, but none differentially expressed ${ }^{75}$. Therefore, TADs can be completely rearranged without causing transcriptional mis-expression, possibly because these translocations did not disconnect enhancers from their target genes. In a second study, Laugsch et al. used human induced pluripotent stem cells (hiPSCs) derived from a branchiooculofacial syndrome (BOFS) patient and differentiated into human neural crest cells (hNCCs) to analyze the effect of an 89 
$\mathrm{Mb}$ inversion overlapping the TFAP2A gene ${ }^{76}$. This inversion prevents TFAP2A from interacting with its cognate enhancers, causing the downregulation of TFP2A's expression. As a consequence of the inversion, the TFP2A enhancers are in proximity to several other genes. This proximity did however not lead to the formation of new chromatin interactions, nor was the expression of these genes affected. Therefore, chromosomal inversions placing genes in proximity to different enhancers do not necessarily cause enhancer adoption ${ }^{76}$, suggesting that enhancer-promoter interaction specificity and/or other mechanisms are involved.

\section{Engineered chromosomal rearrangements}

\section{Deleting CTCF-binding sites at TAD boundaries}

The functional consequences of structural rearrangements during evolution and disease have been very informative on the role of chromatin organization. However, in the case of disease, we are focusing on events that do cause pathologies and might represent exceptions (because they target a particular locus) rather than the rule. As most structural rearrangements have been identified as the cause of a disease, it is expected that they will display functional consequences. On the other hand, evolutionary studies focus on the result of a process where only the fittest solution has been selected. Most inconveniently, in both cases, we do not control the location of the structural rearrangements. To go beyond natural occurrences, the functional role of TADs has recently been investigated using a large variety of engineered chromosomal rearrangements. A first series of experiments evaluated the impact of selectively deleting CTCF-binding sites located at TAD boundaries. Intuitively, one might expect that if TAD boundaries are essential to insulate the regulatory landscapes of two adjacent TADs, deleting a TAD boundary would cause ectopic interactions between adjacent TADs and eventually fuse them. As a result, the expression of nearby genes should be affected. Experimental evidence however suggests that this model might be too simplistic. One of the earliest examples came from the seminal paper describing TADs on a portion of the $X$ chromosome in mouse embryonic stem cells ${ }^{26}$. Deleting a $58 \mathrm{~kb}$-long region at the boundary between the Xist and Tsix TADs induced the formation of ectopic contacts across the deletion site. However, this deletion did not lead to a complete fusion between the two adjacent TADs: their global architecture was maintained and most contacts were still restricted to the original TADs. The effect on gene expression at the locus was however not assessed. The transcriptional effect of deleting or mutating CTCF-binding sites was investigated at other loci. For example, at the $b$-globin locus, the mutation of four nucleotides in a core CTCF-binding site ( $3^{\prime} \mathrm{HS} 1$ ) located downstream of the locus and overlapping a sub-TAD boundary ${ }^{77}$ completely abolished CTCF binding and strongly affected the interaction of $3^{\prime} \mathrm{HS} 1$ with the $b$-globin locus ${ }^{78}$. However, this mutation did not affect the expression of the $b$-globin genes. In addition, the loss of this boundary did not cause the inappropriate activation of other genes (mouse olfactory receptor genes) located across the boundary, as would be expected if the insulating property of the site was lost ${ }^{78}$. Similarly, the deletion of a CTCF-binding site at the Sox2 locus did not affect the expression of most genes in the vicinity, except for the Fbn2 gene which is $\sim 230 \mathrm{~kb}$ away ${ }^{32}$. Thorough dissection of the homeotic homeobox (Hox) gene cluster HoxD confirmed that the simple deletion of a CTCF-binding site is not sufficient to cause TAD fusion. The HoxD cluster is located at a boundary between two TADs, active in the proximal or distal limb buds respectively. This boundary consists of several adjacent CTCFbinding sites whose partial deletion did not significantly affect TAD insulation and gene expression. Only larger deletions, actually deleting a $400 \mathrm{~kb}$ region including the HoxD cluster itself, did result in 
TAD fusion. And yet, despite the formation of this neo-TAD, only minor effects on gene expression were observed, and distally- and proximally-acting enhancers retained their specificity even when placed in the same $T A D^{79,80}$. As at the HoxD locus, TAD boundaries usually consist of a cluster of CTCF-binding sites. The deletion of a single site from this cluster generally has only local effects, the boundary been shifted to the next neighboring CTCF-binding site. In some cases, even large deletions preserve TAD boundaries. For example, deleting a $\sim 82 \mathrm{~kb}$ region encompassing the highly conserved Firre locus, a non-coding RNA located on the $\mathrm{X}$ chromosome that forms a very strong TAD boundary with a cluster of 15 CTCF-binding sites, did not affect boundary formation ${ }^{81}$. This locus might however represent an extreme case. Indeed, the progressive deletion of four, eight, or nine adjacent CTCF-binding sites located at the boundary between the Sox9 and Kcnj2 TADs does eventually result in TAD fusion ${ }^{82}$, as observed at the HoxD locus ${ }^{79,80}$. Again, this fusion has only mild effects on gene expression: the expression pattern of Sox9 remains largely unaffected with only a slight down-regulation in the most severe deletion, and the deletion of eight or nine CTCF-binding sites causes a faint ectopic expression of Kcnj2 in the digit anlagen, mimicking Sox9's expression pattern ${ }^{82}$. Other loci seem however more sensitive to TAD boundary deletions. A particularly extreme example has been reported at the HoxA cluster during motor neuron differentiation ${ }^{83}$. The deletion of a single CTCF-binding site (a nine bp deletion between HoxA5 and HoxA6) results in the loss of the boundary and the expansion of the active chromatin domain into the repressed domain. Surprisingly, this deletion also affects the binding of CTCF to a nearby insulator site (between HoxA6 and HoxA7). The boundary is therefore shifted to a more distal CTCF-binding site (between HoxA7 and HoxA9). In addition, deleting this single CTCF-binding site in the HoxA cluster (as well as the equivalent deletion in the $\mathrm{HoxC}$ cluster) in motor neurons results in homeotic transformations typical of Hox gene mis-regulation ${ }^{84}$. This increased sensitivity to CTCF-binding site deletion might be the consequence of a weaker and more plastic boundary. Indeed, a single CTCF-binding site separates some of the HoxA genes, and the location of this TAD boundary is cell-type specific: located between HoxA5 and HoxA6 in motor neuron, but between HoxA10 and HoxA11 in embryonic stem cells ${ }^{84}$. It is thus possible that weaker TAD boundaries or boundaries between sub-TADs are more sensitive to deletions. In line with this hypothesis, the deletion of CTCF-binding sites at five super-enhancer domains (sub-TADs formed between a super-enhancer and its target genes linked by CTCF-binding sites) caused the down-regulation of genes located within the same domain and the up-regulation of genes located outside the domain. The up-regulated genes formed ectopic chromosomal interactions with regions located across the deleted CTCF-binding site, consistent with a classical insulation role of $\mathrm{CTCF}^{85}$. Similarly, the deletion of the HS-38 and HS-39 CTCF-binding sites delimiting a $\sim 80 \mathrm{~kb}$ sub-TAD at the mouse $\alpha$-globin locus affected the expression of several genes located directly upstream and altered the 3D organization of the locus ${ }^{86,87}$. Finally, an important consideration came from the analysis of deletions and inversions at the HoxD locus: all ectopic contacts observed after the rearrangements involved regions that were already weakly contacted in the wild-type situation ${ }^{88}$. In conclusion, these studies suggest that TADs do favor the formation of local interactions within them, possibly through epigenetic-driven interactions ${ }^{89,90}$, but that their boundaries do not act as extremely strict borders.

The example of the Shh locus

The Sonic hedgehog (Shh) locus in mouse has been the subject of thorough investigations and is therefore a good case study to assess the effect of structural rearrangements on gene expression. Shh has a critical role during development and has been involved in the patterning of several organs 
including the limb. The limb-specific expression of Shh in the posterior limb bud mesenchyme is regulated by the Zone of Polarizing Activity (ZPA) Regulatory Sequence (ZRS) enhancer located about a megabase away within an intron of the unrelated $L M B R 1$ gene $^{91}$. Single-nucleotide mutations in the ZRS affect Shh expression and can either cause preaxial polydactyly in mammals ${ }^{91}$ or be responsible for the absence of limbs in snakes ${ }^{92}$. Shh and its ZRS enhancer are located within the same megabase-sized $T A D^{37}$. Several recent studies ${ }^{93-95}$ used this very well-studied locus to generate deletions and inversions and thus assess their effects on chromatin organization and gene expression (Figure1).

First, the authors generated deletions within the Shh TAD, decreasing the size of the TAD and the linear distance between Shh and the ZRS without affecting TAD boundaries $\left(\Delta 700^{94}\right.$ and DEL $\left.(5-8)^{93}\right)$. Despite a drastic change in the TAD's size, these deletions did not affect the expression of Shh. Second, the authors generated a series of deletions affecting CTCF-binding sites at the Shh TAD boundaries. As above, a single CTCF-binding site deletion had only a minor effect, merely shifting the TAD boundary to the next CTCF-binding site without affecting Shh expression. Surprisingly though, these single CTCF-binding site deletions caused a decrease in the 3D distance between Shh and the ZRS (as assessed by 3D DNA FISH). The expression of Shh was only affected when several adjacent CTCF-binding sites were deleted, as in the $\Delta \mathrm{i} 4: \mathrm{i} 5^{95}$ or $\Delta \mathrm{Lmbr} 1 \mathrm{P}^{94}$ mutants. In addition, the deletion of different neighboring CTCF-binding sites is not equivalent: deleting the two CTCF-binding sites directly surrounding the ZRS is more deleterious than deleting a region encompassing the Lmbr1 promoter. In both cases, the deletion of CTCF-binding sites did not lead to TAD fusion, but rather to the formation of ectopic contacts. On the same note, previous results showed that the deletion of the ZRS enhancer does not prevent the formation of interactions between the surrounding regions and the $S h h$ promoter $^{96}$. Interestingly, in the $\Delta 35$ deletion affecting the promoter of $L m b r 1$, the ZRS can now contact some regions further to the right, including the $M n \times 1$ gene which becomes overexpressed in the limb. This ectopic expression is however specific to the ZRS, as other enhancers located in the same region (MFCS4 and MACS1) do not affect Mnx1 expression. This suggests that enhancer adoption is specific to some enhancer-promoter pairs.

Finally, inversions placing a TAD boundary between Shh and the ZRS did affect the expression of Shh, confirming that TAD boundaries act as insulators. However, this insulation effect becomes milder as the linear distance between Shh and the ZRS decreases. Further characterization of this locus would require monitoring the expression of $S h h$ at the single-cell level and simultaneously measuring the distance between Shh and the ZRS, as recently performed at the even-skipped locus in Drosophila ${ }^{97}$. Indeed, a transient and dynamic interaction between Shh and the ZRS, potentially sufficient to trigger transcription, will be difficult to assess in a bulk heterogeneous population of cells. In addition, the role of cohesin in the organization of the locus should be clarified, as the presence of cohesin in the different mutants could explain their mild effects on gene expression.

\section{Are inversions more deleterious than deletions?}

The example of the Shh TAD suggests that an inversion affecting a TAD boundary has a stronger effect than the deletion of a TAD boundary. This difference has been confirmed at the Sox $9 / K c n j 2^{82}$ and Tfap $2 c / B m p 7^{98}$ loci. However, recent work in Drosophila revealed that genomic inversions do not necessarily strongly affect gene expression ${ }^{99}$. Allele-specific chromatin organization and gene expression were analyzed in heterozygote embryos carrying highly rearranged balancer chromosomes. Despite the presence of thousands of large and small structural variants differentiating the wild-type from the balancer alleles, remarkably little effect on transcription was 
observed: only $10 \%$ of genes were differentially expressed. In particular, the expression of genes located around inversions breakpoints was not systematically affected, suggesting that enhancer adoption might be relatively infrequent. Moreover, the structural variants found in balancer chromosomes are not enriched at TAD boundaries nor in gene-poor regions, but rather distributed randomly. It is likely that several mechanisms such as enhancer redundancy, which is particularly pervasive in Drosophila ${ }^{100}$, might buffer the effect of chromosomal rearrangements. In another study, the analysis of engineered inversions at three Drosophila testis-specific gene clusters ${ }^{101}$ also revealed little impact on the expression of neighboring genes. How can we explain this discrepancy between mammals and flies? First, it is possible that the effect of inversions is highly tissue-specific, and might be missed when analyzed in whole embryos. However, the transcriptional effect of inversions was not stronger in adult fly heads (a more homogeneous cell population largely composed of neurons) than in whole embryos ${ }^{99}$. Besides, inversions affecting three testis-specific gene clusters did not significantly affect gene expression in the testes ${ }^{101}$. Second, the relatively smaller size of regulatory domains in Drosophila (resulting in enhancers located usually closer to their target genes than in vertebrates), and the smaller number of enhancers per gene could reduce the likelihood of observing an inversion breakpoint between an enhancer and its target gene. In addition, fly genomes may be somewhat more plastic than mammalian genomes. Indeed, the synteny of the Hox gene cluster is poorly conserved between several Drosophilids, contrary to mammals where this order is strongly conserved ${ }^{102,103}$. It is also likely that the selection of the loci analyzed in mammals, biased towards important developmental genes or oncogenes, is enriched in regions that are more sensitive to chromosomal inversions. In fact, inversions are not always deleterious in mammals. As mentioned above, chromosomal inversions did not cause transcriptional mis-regulation by enhancer adoption at the TFAP2A locus in human cells ${ }^{76}$. Finally, a potential concern might arise from the selection, in balancer chromosomes, of the least harmful mutations. However, the large number of variants found in the balancer chromosomes compared their wild type counterparts, their nature and location argue against such a strong selection ${ }^{99}$. In addition, similar results were observed with de novo inversions ${ }^{101}$.

We therefore propose that at many (maybe most) loci, chromosomal inversions can be buffered by two mechanisms (Figure 2): First, disrupting the interaction between an enhancer and its target gene(s) by placing a TAD boundary in between can be compensated by the presence of one or multiple other redundant enhancers ${ }^{100,104,105}$. Such redundant or "shadow" enhancers drive the same, or a similar, expression pattern. Their deletion (or relocation to a different TAD) would therefore not cause a visible phenotype ${ }^{106-108}$. This compensatory mechanism can sustain a nearly normal expression level of the target gene. Second, while enhancer adoption is possible at some loci, it depends on parameters, such as enhancer-promoter specificity ${ }^{15,109}$, still poorly understood. Placing an enhancer in a new regulatory domain will thus not necessarily create ectopic contacts and affect the expression of the genes located in that domain. In line with this hypothesis, a series of inversions at the Epha4 locus in mouse did not affect the expression of all nearby genes to the same extent, and the most strongly mis-regulated gene was not necessarily the one directly adjacent to the inversion breakpoint ${ }^{33}$.

\section{Conclusion/Perspective}

Despite years of research, the functional role of TADs remains unclear. TADs have been proposed to influence gene expression through two complementary mechanisms. On one hand, they can act as a 
barrier to prevent unwanted interactions with regions located outside the domain. On the other hand, they can promote interactions between regions located within them. While these two activities can be observed at some loci, recent genomic engineering experiments suggest a more subtle function. At short distance, TAD boundaries are not required to promote intra-TAD interactions, but they become more crucial across large distances. Similarly, TAD boundaries do not act as strong borders, as their deletion only marginally affects the insulation of the domains. Instead, TAD boundaries might be important to provide precision and robustness to gene expression ${ }^{82}$. Overall, the organization of the genome in TADs is clearly an important and functional feature of the genome, but the strength of their boundaries seems to be largely locus-specific, if not condition- and species-specific. The discrepancy observed between studies assessing the functional consequences of TAD boundary modifications could indeed reflect biological differences. For example, it could be linked to differences in regulatory landscapes, with genes regulated by enhancers displaying different characteristics (distance, specificity, activity, redundancy, etc...). But this discrepancy might also to some extent simply be due to the poor definition of TADs. Finally, it is important to keep in mind that most available studies rely on bulk $\mathrm{Hi}-\mathrm{C}$ data to define TAD boundaries. Indeed, recent single-cell $\mathrm{Hi}-\mathrm{C}$ and imaging experiments uncovered extensive heterogeneity in TAD boundaries between conditions and cell-types at the single-cell level ${ }^{18,19,110,111}$. This further supports the fact that other mechanisms beside chromatin organization into TADs are driving appropriate gene expression. Clearly, clarifying the link between chromatin organization and transcription regulation will require further investigations. These should in particular aim at better characterizing the rules governing enhancer-promoter interactions specificity, for example through the thorough dissection of specific loci and the precise live monitoring of chromatin organization and gene expression in single cells.

\section{Acknowledgments}

I thank all the members of the Ghavi-Helm laboratory, Daniel Jost and Kiran Padmanabhan for useful comments. Work in YGH's laboratory is funded by an ERC starting grant (759708) and the Ecole Normale Supérieure de Lyon.

\section{Reference}

1. Banerji, J., Rusconi, S. \& Schaffner, W. Expression of a $\beta$-globin gene is enhanced by remote SV40 DNA sequences. Cell 27, 299-308 (1981).

2. Moreau, P. et al. The SV40 72 base repair repeat has a striking effect on gene expression both in SV40 and other chimeric recombinants. Nucleic Acids Res 9, 6047-6068 (1981).

3. Gross, D. S. \& Garrard, W. T. Nuclease Hypersensitive Sites in Chromatin. Annual Review of Biochemistry 57, 159-197 (1988).

4. Creyghton, M. P. et al. Histone H3K27ac separates active from poised enhancers and predicts developmental state. Proc Natl Acad Sci USA 107, 21931 (2010).

5. Heintzman, N. D. et al. Histone modifications at human enhancers reflect global cell-type-specific gene expression. Nature 459, 108-112 (2009).

6. Rada-Iglesias, A. et al. A unique chromatin signature uncovers early developmental enhancers in humans. Nature 470, 279-283 (2011).

7. Zentner, G. E., Tesar, P. J. \& Scacheri, P. C. Epigenetic signatures distinguish multiple classes of enhancers with distinct cellular functions. Genome Res 21, 1273-1283 (2011).

8. Bonn, S. et al. Tissue-specific analysis of chromatin state identifies temporal signatures of enhancer activity during embryonic development. Nat Genet 44, 148-156 (2012).

9. Long, H. K., Prescott, S. L. \& Wysocka, J. Ever-Changing Landscapes: Transcriptional Enhancers in Development and Evolution. Cell 167, 1170-1187 (2016). 
10. Ghavi-Helm, Y. et al. Enhancer loops appear stable during development and are associated with paused polymerase. Nature 512, 96-100 (2014).

11. de Laat, W. \& Duboule, D. Topology of mammalian developmental enhancers and their regulatory landscapes. Nature 502, 499-506 (2013).

12. Dixon, J. R. et al. Topological domains in mammalian genomes identified by analysis of chromatin interactions. Nature 485, 376-380 (2012).

13. Andrey, G. et al. Characterization of hundreds of regulatory landscapes in developing limbs reveals two regimes of chromatin folding. Genome Res. 27, 223-233 (2017).

14. Sanyal, A., Lajoie, B. R., Jain, G. \& Dekker, J. The long-range interaction landscape of gene promoters. Nature 489, 109-113 (2012).

15. van Arensbergen, J., van Steensel, B. \& Bussemaker, H. J. In search of the determinants of enhancer-promoter interaction specificity. Trends in Cell Biology 24, 695-702 (2014).

16. Giorgetti, L. \& Heard, E. Closing the loop: 3C versus DNA FISH. Genome Biology 17, (2016).

17. Lieberman-Aiden, E. et al. Comprehensive Mapping of Long-Range Interactions Reveals Folding Principles of the Human Genome. Science 326, 289-293 (2009).

18. Finn, E. H. et al. Extensive Heterogeneity and Intrinsic Variation in Spatial Genome Organization. Cell 176, 1502-1515.e10 (2019).

19. Bintu, B. et al. Super-resolution chromatin tracing reveals domains and cooperative interactions in single cells. Science 362, eaau1783 (2018).

20. Boettiger, A. N. et al. Super-resolution imaging reveals distinct chromatin folding for different epigenetic states. Nature 529, 418-422 (2016).

21. Mateo, L. J. et al. Visualizing DNA folding and RNA in embryos at single-cell resolution. Nature 568, 49-54 (2019).

22. Cardozo Gizzi, A. M. et al. Microscopy-Based Chromosome Conformation Capture Enables Simultaneous Visualization of Genome Organization and Transcription in Intact Organisms. Molecular Cell 74, 212-222.e5 (2019).

23. Guelen, L. et al. Domain organization of human chromosomes revealed by mapping of nuclear lamina interactions. Nature 453, 948-951 (2008).

24. Rao, S. S. P. et al. A 3D Map of the Human Genome at Kilobase Resolution Reveals Principles of Chromatin Looping. Cell 159, 1665-1680 (2014).

25. Sexton, T. et al. Three-Dimensional Folding and Functional Organization Principles of the Drosophila Genome. Cell 148, 458-472 (2012).

26. Nora, E. P. et al. Spatial partitioning of the regulatory landscape of the $\mathrm{X}$-inactivation centre. Nature 485, 381-385 (2012).

27. Phillips-Cremins, J. E. et al. Architectural Protein Subclasses Shape 3D Organization of Genomes during Lineage Commitment. Cell 153, 1281-1295 (2013).

28. Sanborn, A. L. et al. Chromatin extrusion explains key features of loop and domain formation in wild-type and engineered genomes. Proc Natl Acad Sci USA 112, E6456 (2015).

29. Fudenberg, G. et al. Formation of Chromosomal Domains by Loop Extrusion. Cell Reports 15, 2038-2049 (2016).

30. Fudenberg, G., Abdennur, N., Imakaev, M., Goloborodko, A. \& Mirny, L. A. Emerging

Evidence of Chromosome Folding by Loop Extrusion. Cold Spring Harb Symp Quant Biol 82, 45-55 (2017).

31. Guo, Y. et al. CRISPR Inversion of CTCF Sites Alters Genome Topology and Enhancer/Promoter Function. Cell 162, 900-910 (2015).

32. de Wit, E. et al. CTCF Binding Polarity Determines Chromatin Looping. Molecular Cell 60, 676-684 (2015).

33. Kraft, K. et al. Serial genomic inversions induce tissue-specific architectural stripes, gene misexpression and congenital malformations. Nature Cell Biology 21, 305 (2019).

34. Nora, E. P. et al. Targeted Degradation of CTCF Decouples Local Insulation of Chromosome Domains from Genomic Compartmentalization. Cell 169, 930-944.e22 (2017). 
35. Forcato, M. et al. Comparison of computational methods for Hi-C data analysis. Nat Methods 14, 679-685 (2017).

36. Zufferey, M., Tavernari, D., Oricchio, E. \& Ciriello, G. Comparison of computational methods for the identification of topologically associating domains. Genome Biology 19, 217 (2018).

37. Symmons, O. et al. Functional and topological characteristics of mammalian regulatory domains. Genome Res. 24, 390-400 (2014).

38. Nora, E. P. et al. Spatial partitioning of the regulatory landscape of the $\mathrm{X}$-inactivation centre. Nature 485, 381-385 (2012).

39. Zhan, Y. et al. Reciprocal insulation analysis of $\mathrm{Hi}-\mathrm{C}$ data shows that TADs represent a functionally but not structurally privileged scale in the hierarchical folding of chromosomes. Genome Res 27, 479-490 (2017).

40. Le Dily, F. et al. Distinct structural transitions of chromatin topological domains correlate with coordinated hormone-induced gene regulation. Genes Dev 28, 2151-2162 (2014).

41. Shen, Y. et al. A map of the cis-regulatory sequences in the mouse genome. Nature 488, 116-120 (2012).

42. Delaneau, O. et al. Chromatin three-dimensional interactions mediate genetic effects on gene expression. Science 364, eaat8266 (2019).

43. Schwarzer, W. et al. Two independent modes of chromatin organization revealed by cohesin removal. Nature 551, 51-56 (2017).

44. Cuartero, S. et al. Control of inducible gene expression links cohesin to hematopoietic progenitor self-renewal and differentiation. Nature Immunology 19, 932 (2018).

45. Krefting, J., Andrade-Navarro, M. A. \& Ibn-Salem, J. Evolutionary stability of topologically associating domains is associated with conserved gene regulation. BMC Biology 16, 87 (2018).

46. Vietri Rudan, M. et al. Comparative Hi-C Reveals that CTCF Underlies Evolution of Chromosomal Domain Architecture. Cell Reports 10, 1297-1309 (2015).

47. Harmston, N. et al. Topologically associating domains are ancient features that coincide with Metazoan clusters of extreme noncoding conservation. Nature Communications 8, 441 (2017).

48. Renschler, G. et al. Hi-C guided assemblies reveal conserved regulatory topologies on $\mathrm{X}$ and autosomes despite extensive genome shuffling. bioRxiv 580969 (2019). doi:10.1101/580969

49. Lazar, N. H. et al. Epigenetic maintenance of topological domains in the highly rearranged gibbon genome. Genome Res. 28, 983-997 (2018).

50. Canela, A. et al. Genome Organization Drives Chromosome Fragility. Cell 170, 507-521.e18 (2017).

51. Aymard, F. et al. Genome-wide mapping of long-range contacts unveils clustering of DNA double-strand breaks at damaged active genes. Nature Structural \& Molecular Biology 24, 353361 (2017).

52. Zhang, Y. et al. Spatial Organization of the Mouse Genome and Its Role in Recurrent Chromosomal Translocations. Cell 148, 908-921 (2012).

53. Engreitz, J. M., Agarwala, V. \& Mirny, L. A. Three-Dimensional Genome Architecture Influences Partner Selection for Chromosomal Translocations in Human Disease. PLOS ONE 7, e44196 (2012).

54. Berthelot, C., Muffato, M., Abecassis, J. \& Roest Crollius, H. The 3D Organization of Chromatin Explains Evolutionary Fragile Genomic Regions. Cell Reports 10, 1913-1924 (2015).

55. Roix, J. J., McQueen, P. G., Munson, P. J., Parada, L. A. \& Misteli, T. Spatial proximity of translocation-prone gene loci in human lymphomas. Nature Genetics 34, 287-291 (2003).

56. Fudenberg, G. \& Pollard, K. S. Chromatin features constrain structural variation across evolutionary timescales. PNAS 116, 2175-2180 (2019).

57. Lupiáñez, D. G., Spielmann, M. \& Mundlos, S. Breaking TADs: How Alterations of Chromatin Domains Result in Disease. Trends in Genetics 32, 225-237 (2016).

58. Spielmann, M. \& Mundlos, S. Structural variations, the regulatory landscape of the genome and their alteration in human disease. BioEssays 35, 533-543 
59. Spielmann, M., Lupiáñez, D. G. \& Mundlos, S. Structural variation in the 3D genome. Nature Reviews Genetics 19, 453-467 (2018).

60. Kaiser, V. B. \& Semple, C. A. When TADs go bad: chromatin structure and nuclear organisation in human disease. F1000Res 6, (2017).

61. Lupiáñez, D. G. et al. Disruptions of Topological Chromatin Domains Cause Pathogenic Rewiring of Gene-Enhancer Interactions. Cell 161, 1012-1025 (2015).

62. Giorgio, E. et al. A large genomic deletion leads to enhancer adoption by the lamin B1 gene: a second path to autosomal dominant adult-onset demyelinating leukodystrophy (ADLD). Hum Mol Genet 24, 3143-3154 (2015).

63. Flöttmann, R. et al. Microdeletions on 6 p22.3 are associated with mesomelic dysplasia Savarirayan type. Journal of Medical Genetics 52, 476-483 (2015).

64. Spielmann, M. et al. Homeotic Arm-to-Leg Transformation Associated with Genomic Rearrangements at the PITX1 Locus. The American Journal of Human Genetics 91, 629-635 (2012).

65. Redin, C. et al. The genomic landscape of balanced cytogenetic abnormalities associated with human congenital anomalies. Nature Genetics 49, 36-45 (2017).

66. Franke, M. et al. Formation of new chromatin domains determines pathogenicity of genomic duplications. Nature 538, 265-269 (2016).

67. Lettice, L. A. et al. Enhancer-adoption as a mechanism of human developmental disease. Human Mutation 32, 1492-1499

68. Northcott, P. A. et al. Enhancer hijacking activates GFI1 family oncogenes in medulloblastoma. Nature 511, 428-434 (2014).

69. Ibn-Salem, J. et al. Deletions of chromosomal regulatory boundaries are associated with congenital disease. Genome Biology 15, 423 (2014).

70. Northcott, P. A. et al. The whole-genome landscape of medulloblastoma subtypes. Nature 547, 311-317 (2017).

71. Flavahan, W. A. et al. Insulator dysfunction and oncogene activation in IDH mutant gliomas. Nature 529, 110-114 (2016).

72. Weischenfeldt, J. et al. Pan-cancer analysis of somatic copy-number alterations implicates IRS4 and IGF2 in enhancer hijacking. Nature Genetics 49, 65-74 (2017).

73. Hnisz, D. et al. Activation of proto-oncogenes by disruption of chromosome neighborhoods. Science 351, 1454-1458 (2016).

74. Sun, J. H. et al. Disease-Associated Short Tandem Repeats Co-localize with Chromatin Domain Boundaries. Cell 175, 224-238.e15 (2018).

75. Dong, Z. et al. Identification of balanced chromosomal rearrangements previously unknown among participants in the 1000 Genomes Project: implications for interpretation of structural variation in genomes and the future of clinical cytogenetics. Genetics in Medicine 20, 697 (2018).

76. Laugsch, M. et al. Modeling the Pathological Long-Range Regulatory Effects of Human Structural Variation with Patient-Specific hiPSCs. Cell Stem Cell (2019). doi:10.1016/j.stem.2019.03.004

77. Huang, P. et al. Comparative analysis of three-dimensional chromosomal architecture identifies a novel fetal hemoglobin regulatory element. Genes Dev. 31, 1704-1713 (2017).

78. Splinter, E. et al. CTCF mediates long-range chromatin looping and local histone modification in the $\beta$-globin locus. Genes Dev. 20, 2349-2354 (2006).

79. Rodríguez-Carballo, E. et al. The HoxD cluster is a dynamic and resilient TAD boundary controlling the segregation of antagonistic regulatory landscapes. Genes Dev. 31, 2264-2281 (2017).

80. Rodríguez-Carballo, E., Lopez-Delisle, L., Yakushiji-Kaminatsui, N., Ullate-Agote, A. \& Duboule, D. Impact of genome architecture on the functional activation and repression of Hox regulatory landscapes. BMC Biol 17, (2019). 
81. Barutcu, A. R., Maass, P. G., Lewandowski, J. P., Weiner, C. L. \& Rinn, J. L. A TAD boundary is preserved upon deletion of the CTCF-rich Firre locus. Nature Communications 9, 1444 (2018).

82. Despang, A. et al. Functional dissection of the Sox9-Kcnj2 locus identifies nonessential and instructive roles of TAD architecture. Nat Genet 51, 1263-1271 (2019).

83. Narendra, V. et al. CTCF establishes discrete functional chromatin domains at the Hox clusters during differentiation. Science 347, 1017-1021 (2015).

84. Narendra, V., Bulajić, M., Dekker, J., Mazzoni, E. O. \& Reinberg, D. CTCF-mediated topological boundaries during development foster appropriate gene regulation. Genes Dev. 30, 2657-2662 (2016).

85. Dowen, J. M. et al. Control of Cell Identity Genes Occurs in Insulated Neighborhoods in Mammalian Chromosomes. Cell 159, 374-387 (2014).

86. Hanssen, L. L. P. et al. Tissue-specific CTCF-cohesin-mediated chromatin architecture delimits enhancer interactions and function in vivo. Nature Cell Biology 19, 952-961 (2017).

87. Oudelaar, A. M. et al. A revised model for promoter competition based on multi-way chromatin interactions. (Genomics, 2019). doi:10.1101/612275

88. Fabre, P. J. et al. Large scale genomic reorganization of topological domains at the HoxD locus. Genome Biol 18, (2017).

89. Ulianov, S. V. et al. Active chromatin and transcription play a key role in chromosome partitioning into topologically associating domains. Genome Res. 26, 70-84 (2016).

90. Ghosh, S. K. \& Jost, D. How epigenome drives chromatin folding and dynamics, insights from efficient coarse-grained models of chromosomes. PLoS Comput Biol 14, (2018).

91. Lettice, L. A. et al. A long-range Shh enhancer regulates expression in the developing limb and fin and is associated with preaxial polydactyly. Hum Mol Genet 12, 1725-1735 (2003).

92. Kvon, E. Z. et al. Progressive Loss of Function in a Limb Enhancer during Snake Evolution. Cell 167, 633-642.e11 (2016).

93. Symmons, O. et al. The Shh Topological Domain Facilitates the Action of Remote Enhancers by Reducing the Effects of Genomic Distances. Developmental Cell 39, 529-543 (2016).

94. Williamson, I. et al. Developmentally regulated Shh expression is robust to TAD perturbations. bioRxiv 609941 (2019). doi:10.1101/609941

95. Paliou, C. et al. Preformed chromatin topology assists transcriptional robustness of Shh during limb development. PNAS 201900672 (2019). doi:10.1073/pnas.1900672116

96. Amano, T. et al. Chromosomal Dynamics at the Shh Locus: Limb Bud-Specific Differential Regulation of Competence and Active Transcription. Developmental Cell 16, 47-57 (2009).

97. Chen, H. et al. Dynamic interplay between enhancer-promoter topology and gene activity. Nat Genet 50, 1296-1303 (2018).

98. Tsujimura, T. et al. A Discrete Transition Zone Organizes the Topological and Regulatory Autonomy of the Adjacent Tfap2c and Bmp7 Genes. PLOS Genetics 11, e1004897 (2015).

99. Ghavi-Helm, Y. et al. Highly rearranged chromosomes reveal uncoupling between genome topology and gene expression. Nat Genet 51, 1272-1282 (2019).

100. Cannavò, E. et al. Shadow Enhancers Are Pervasive Features of Developmental Regulatory Networks. Current Biology 26, 38-51 (2016).

101. Meadows, L. A., Chan, Y. S., Roote, J. \& Russell, S. Neighbourhood Continuity Is Not Required for Correct Testis Gene Expression in Drosophila. PLOS Biology 8, e1000552 (2010).

102. Negre, B. \& Ruiz, A. HOM-C evolution in Drosophila: is there a need for Hox gene clustering? Trends in Genetics 23, 55-59 (2007).

103. Drosophila 12 Genomes Consortium. Evolution of genes and genomes on the Drosophila phylogeny. Nature 450, 203-218 (2007).

104. Frankel, N. et al. Phenotypic robustness conferred by apparently redundant transcriptional enhancers. Nature 466, 490-493 (2010).

105. Hong, J.-W., Hendrix, D. A. \& Levine, M. S. Shadow Enhancers as a Source of Evolutionary Novelty. Science 321, 1314-1314 (2008). 
106. Cretekos, C. J. et al. Regulatory divergence modifies limb length between mammals. Genes Dev. 22, 141-151 (2008).

107. Hay, D. et al. Genetic dissection of the $\alpha$-globin super-enhancer in vivo. Nature Genetics 48, 895-903 (2016).

108. Osterwalder, M. et al. Enhancer redundancy provides phenotypic robustness in mammalian development. Nature 554, 239-243 (2018).

109. Zabidi, M. A. et al. Enhancer-core-promoter specificity separates developmental and housekeeping gene regulation. Nature 518, 556-559 (2015).

110. Flyamer, I. M. et al. Single-nucleus $\mathrm{Hi}-\mathrm{C}$ reveals unique chromatin reorganization at oocyteto-zygote transition. Nature 544, 110-114 (2017).

111. Nagano, T. et al. Single-cell Hi-C reveals cell-to-cell variability in chromosome structure. Nature 502, 59-64 (2013).

\section{Figure legends}

Figure 1: Effect of chromosomal rearrangements at the Shh locus.

A. Schematic representation of the mouse $S h h$ locus displaying the location of the relevant genes (black), CTCF-binding sites (red) and of the ZRS. The approximate location of deletions ( $\triangle$ Lmbr1P, $\Delta 35, \Delta 700, \mathrm{DEL}(5-8)$ ) and inversions (INV(-500-C1), INV(6-C2), INV(4-C2), INV(2-C2)) assessed by Symmons et al. ${ }^{93}$, Paliou et $a l^{95}$, and Williamson et al. ${ }^{94}$ is indicated. B. Table summarizing the effect of the deletions and inversions represented in $(A)$ on chromatin organization, 3D distance between Shh and the ZRS, Shh expression, and final phenotype.

Figure2: Schematic representation of a chromosomal inversion affecting a TAD boundary. A chromosomal inversion can shift the location of an enhancer (purple box) from one TAD (orange triangle) to the next (pink triangle). The expression of the genes (green/red rectangles for transcriptionally active or inactive respectively) located in the pink TAD will be affected if their promoter is compatible with the inverted enhancer. The expression of the genes located in the orange TAD might not be affected if other redundant enhancers are located within the same TAD. 


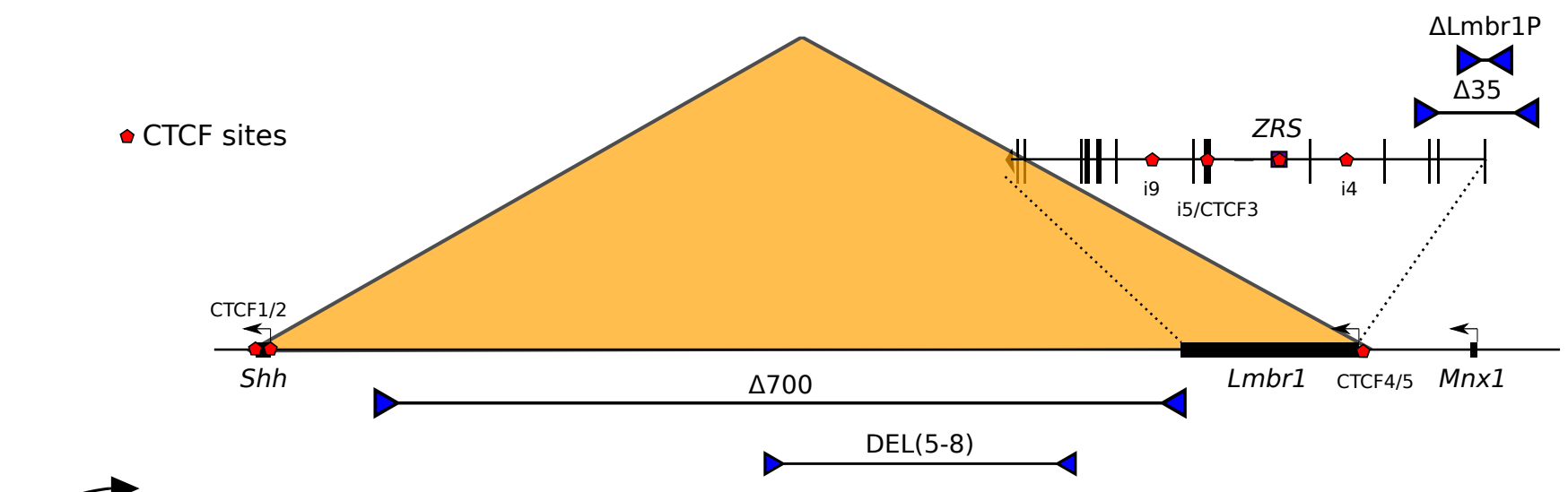

INV(-500-C1)

INV(6-C2)

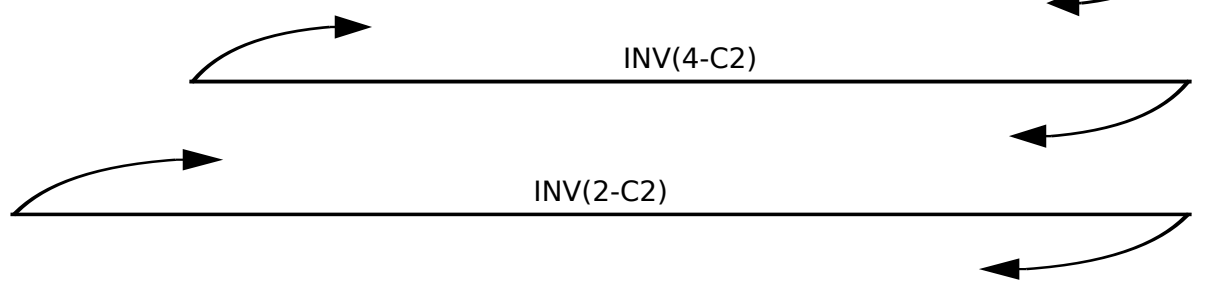

B

\begin{tabular}{|c|c|c|c|c|}
\hline & $\begin{array}{l}\text { Effect on chromatin } \\
\text { organisation }\end{array}$ & $\begin{array}{l}\text { Increased 3D distance } \\
\text { between Shh and ZRS }\end{array}$ & Effect on Shh expression & Phenotype \\
\hline \multirow{2}{*}{$\begin{array}{c}\Delta 700 \\
\operatorname{DEL}(5-8)\end{array}$} & None & NA & No & No \\
\hline & NA & NA & No & No \\
\hline$\triangle$ CTCF1 & shifts boundary to CTCF2 & Yes & No & No \\
\hline$\triangle \mathrm{CTCF2}$ & $\begin{array}{l}\text { some ectopic contacts and } \\
\text { intra-TAD reorganization }\end{array}$ & Yes & No & No \\
\hline$\triangle \mathrm{CTCF3}$ & some intra-TAD reorganization & Yes & No & No \\
\hline$\triangle$ CTCF4 & $\begin{array}{l}\text { increased intra-TAD } \\
\text { interactions }\end{array}$ & No & No & No \\
\hline$\triangle$ CTCF5 & $\begin{array}{l}\text { decreased interactions with } \\
\text { Mnx1 TAD }\end{array}$ & No & No & No \\
\hline$\Delta$ Lmbr1P & shifts boundary to the left (i9) & $\overline{N A}$ & Yes $-20 \%$ reduction & No \\
\hline$\Delta \mathrm{i} 4: \mathrm{i5}$ & $\begin{array}{l}\text { Loss of interation between Shh } \\
\text { and the } L m b r 1 \text { boundary, and } \\
\text { increased interactions with the } \\
\text { Mnx1 TAD }\end{array}$ & Yes & $\begin{array}{c}\text { Yes }-51 \% \text { reduction } \\
98 \% \text { in sensitized background }\end{array}$ & $\begin{array}{l}\text { No, only in sensitized } \\
\text { background }\end{array}$ \\
\hline$\Delta \mathrm{i} 4: \mathrm{i5}: \mathrm{ZRS}$ & $\begin{array}{l}\text { Loss of interation between Shh } \\
\text { and the Lmbr1 boundary, and } \\
\text { increased interactions with the } \\
\text { Mnx1 TAD }\end{array}$ & NA & Yes $-52 \%$ reduction & No \\
\hline INV(-500-C1) & NA & NA & Yes & $\begin{array}{l}\text { Yes in a sensitized } \\
\text { background }\end{array}$ \\
\hline INV(6-C2) & $\begin{array}{l}\text { lost interaction between ZRS } \\
\text { and Shh }\end{array}$ & NA & Yes & $\begin{array}{l}\text { Yes in a sensitized } \\
\text { background }\end{array}$ \\
\hline INV(4-C2) & NA & NA & NA & Yes \\
\hline $\operatorname{INV}(2-C 2)$ & NA & NA & NA & $\begin{array}{l}\text { Yes, but progressively } \\
\text { milder as the distance } \\
\text { between Shh and the } \\
\text { ZRS decreases }\end{array}$ \\
\hline
\end{tabular}



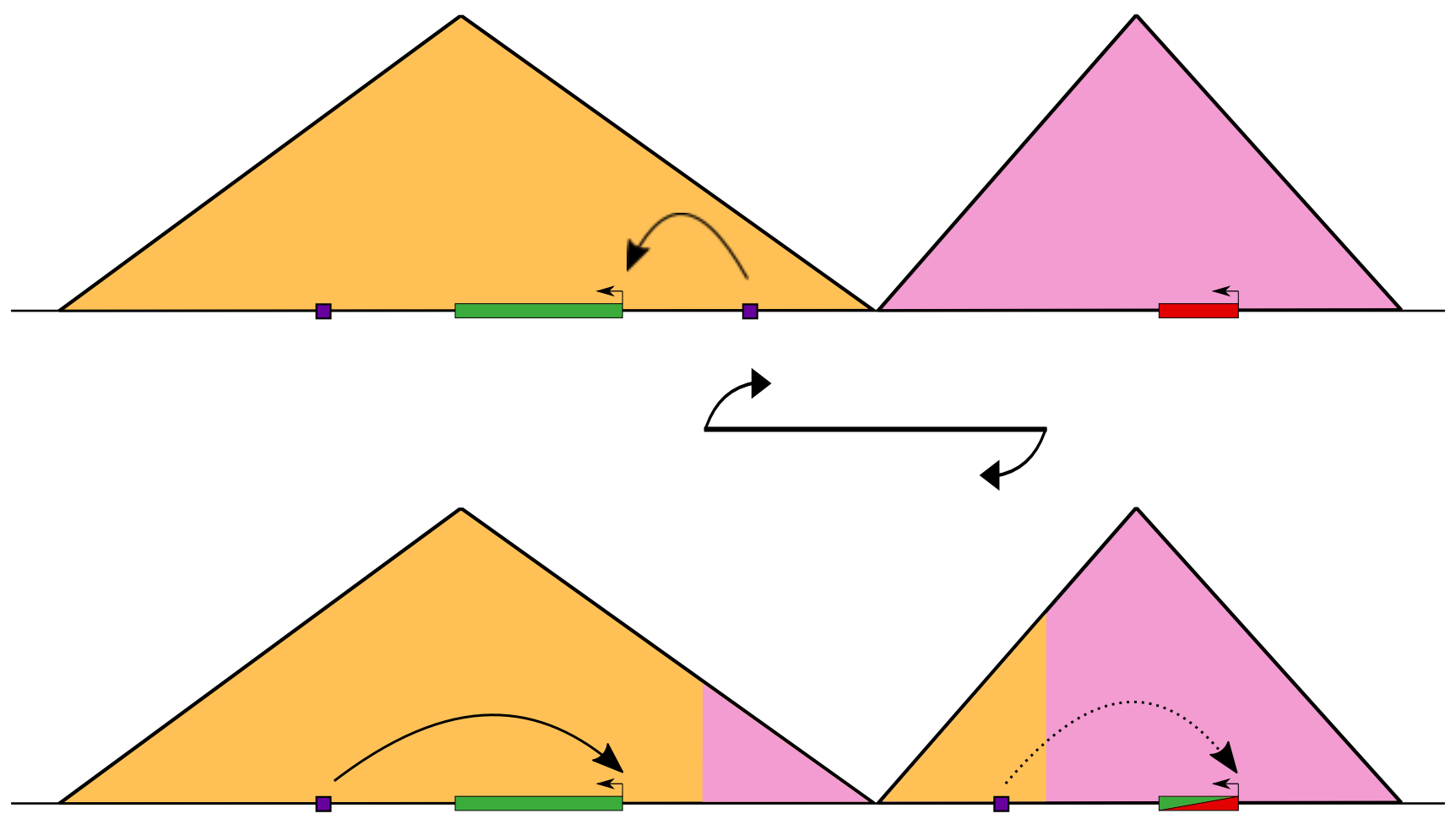

buffering by enhancer redundancy

enhancer adoption only if compatibility 